\title{
Krankenhaus: Impulse aus Dänemark für Deutschland
}

ELKE BERGER, REINHARD BUSSE, BENJAMIN FINGER, KLAUS FOCKE,

ALEXANDER GEISSLER

Elke Berger ist Wissenschaftliche Mitarbeiterin im Fachgebiet Management im Gesundheitswesen an der TU Berlin

Prof. Dr. med. Reinhard Busse ist Leiter des Fachgebiets Management im Gesundheitswesen an der TU Berlin und Co-Director des European Observatory on Health Systems and Policies

Benjamin Finger ist Referent in der Abteilung Versorgungsmanagement beim BKK Dachverband e. V., Berlin

Klaus Focke ist Leiter der Abteilung Versorgungsmanagement beim BKK Dachverband e. V., Berlin

Dr. Alexander Geissler ist Wissenschaftlicher Mitarbeiter im Fachgebiet Management im Gesundheitswesen an der TU Berlin

\author{
In Anbetracht des vorherrschenden Reformbedarfs in \\ der deutschen Krankenhauslandschaft widmet sich \\ der Beitrag der dänischen Krankenhausstrukturreform, \\ die von vielen als mögliches Vorbild, von anderen \\ aber als nicht übertragbar gesehen wird. Hintergrund, \\ Umsetzung und die daraus resultierenden positiven \\ Ergebnisse der dänischen Reform werden dargestellt. \\ Es wird deutlich, warum das dänische Modell ein \\ guter Impulsgeber für Deutschland sein kann. \\ Abschließend wird die Frage der Übertragbarkeit \\ aufgeworfen und beschrieben, wie diese in einem \\ Forschungsvorhaben beantwortet werden soll
}

\section{Hintergrund}

Der deutsche Krankenhaussektor ist mit knapp 1,2 Mio. Beschäftigten in 1951 Krankenhäusern, in denen zuletzt fast 20 Mio. Patienten jährlich behandelt wurden, eine der größten Branchen Deutschlands. Im Jahr 2016 betrugen die Ausgaben für alle stationären und teilstationären Leistungen in Krankenhäusern nahezu 88 Mrd. Euro - ein Betrag, der fast drei Prozent des Bruttoinlandsprodukts entspricht (Statistisches Bundesamt 2016a \& 2016b). Angesichts dessen muss der Veränderungsdruck im Bereich der stationären Versorgung, welcher in den vergangenen Jahren stetig zugenommen hat, nachhaltig abgebaut werden. Zwar sind die Einzelfallkosten der Krankenhäuser aus internationaler Perspektive vergleichsweise niedrig (OECD 2017), aber die kontinuierlich steigende Inanspruchnahme - begünstigt durch Überkapazitäten und abnehmende Verweildauern - lässt die Gesamtkosten konstant steigen (Geissler et al. 2010), ohne dabei eine deutliche Qualitätsverbesserung erkennen zu lassen (Nimptsch \& Mansky 2017). Daher werden vermehrt die derzeitigen Krankenhausstrukturen in Frage gestellt. Obwohl diese häufiger in Anspruch genommen werden als im internationalen Vergleich, sind sie in wesentlichen Teilen mit schlechteren Outcomes assoziiert (Busse et al. 2016).

Geht es um Strukturveränderungen im Krankenhausbereich, ist das sogenannte „dänische Modell“ derzeit hierzulande in aller Munde und wurde in den letzten Jahren verstärkt diskutiert (Augurzky et al. 2014 \& 2015; Busse et al. 2016). Dort wird die stationäre bzw. die sektorenübergreifende Versorgung in einem seit Anfang der 2000er Jahre fortlaufenden Prozess grundlegend neu strukturiert. So wurden u. a. Krankenhäuser neu errichtet, viele kleinere Häuser geschlossen oder umgewidmet, zentrale Notaufnahmen eingerichtet und insgesamt die Zahl der Standorte mit Notaufnahmen halbiert. Begleitet wurden diese Maßnahmen von weitreichenden Qualitätssicherungsmaßnahmen. Die dänische Vorgehensweise ist insofern von besonderem Interesse, als die Struktur- und Steuerungsprobleme der Versorgung vor der Konsolidierung vielfach vergleichbar mit denen in Deutschland waren und zudem aktuellste Evaluationsergebnisse für den Erfolg 
der Reformen sprechen (Christiansen \& Vrangbaek 2018).

Bei allen Ähnlichkeiten mit der deutschen Krankenhausversorgung gibt es doch wesentliche Unterschiede in der Ausgestaltung der Gesundheitssysteme: Im Gegensatz zum Konzept der Sozialversicherung in Deutschland erfolgt etwa die Finanzierung der Gesundheitsversorgung in Dänemark über Steuern (Olejaz et al. 2012). Zudem war der dänische akute Krankenhaussektor bereits vor der

\section{Die dänische Bevölkerung wird heute schneller, mit höherem Personaleinsatz und besserer Qualität als zur Jahrtausendwende versorgt.}

Empfehlungen auf dem Tisch: Da die 16 Kreise bzw. kreisfreien Städte als zu klein für Planung und Durchführung der stationären Gesundheitsversorgung angesehen wurden, waren diese am stärksten von der Strukturreform betroffen: aus 16 wurden 5 neue Regionen, die seit Inkrafttreten der Strukturreform im Januar 2007 für die stationäre Versorgung zuständig sind. Die Verantwortung für die Primärversorgung hingegen liegt bei den Kommunen, deren Zahl von 271 auf 98 reduziert wurde. Für die Gesundheitsversorgung wird jetzt eine Art Gesundheitssteuer erhoben, die der Staat auf Kommunen und Regionen verteilt. Die Regionen finanzieren daraus $80 \%$ der Kosten der stationären Versorgung, während die Kommunen für "ihre" Patienten 20\% beitragen (ebd.).

Die Krankenhausversor-

Strukturreform kleiner, stärker zentralisiert und im Gegensatz zur deutschen Trägerpluralität im Wesentlichen durch die regionalen Verwaltungen geprägt, denen die Krankenhäuser zugordnet sind. Nachdem bereits seit den 1980er Jahren zahlreiche kleinere Krankenhäuser geschlossen wurden, gab es 1997 noch 82 Krankenhäuser mit durchschnittlich 272 Betten, insgesamt also rund 22.000 Betten - übertragen auf Deutschland wären das unter Berücksichtigung der Bevölkerungszahl etwa 1300 Krankenhäuser mit rund 350.000 Betten (Christiansen \& Vrangbaek 2018). Bis 2007 fiel die Zahl der Krankenhäuser weiter bis auf rund 50, was z. T. durch den freiwilligen Konzentrationsprozess bedingt war, der durch die Debatte um eine große Strukturreform ausgelöst wurde. Diese Debatte wurde 2002 durch die damalige liberale Regierung angestoßen, die eine Kommission zu einer umfassenden territorialfunktional-finanziellen Reform der mittleren und unteren Ebene der Verwaltungsstruktur eingesetzt hatte. Zu jener Zeit oblag die Gesundheitsversorgung den 13 Kreisen und drei kreisfreien Städten, die diese alle auch (teilweise) durch örtliche Steuern finanzierten. 2004 lagen die zum damaligen Zeitpunkt auch klar, dass eine solche Restrukturierung der stationären Versorgung zumeist nicht mit den oftmals aus den 1970er Jahren stammenden Krankhausbauten zu erreichen sein wird. Deshalb reifte auch die Idee, einen Fonds für ein umfassendes Investitionsprogramm aufzulegen und $\mathrm{zu}$ $60 \%$ zentral zu finanzieren. Politisch war dabei erst von der Summe von „100 Milliarden“ (dänische Kronen, d. h. ca. 13 Mrd. €) die Rede. Schließlich wurde ein Viertel davon, also 25 Milliarden Kronen (ca. 3,3 Mrd. €) vorgesehen. Dafür wurde ein „Qualitätsfonds“ eingerichtet, worauf sich die Regionen mit ihren Konzepten 2008 bewerben konnten. Zur Evaluation und Begutachtung der Konzepte wurde eine 5-köpfige internationale Kommission eingerichtet und das Fördervolumen auf 40 Milliarden Kronen (ca. 5,5 Mrd. €) erhöht, die bis 2024 in insgesamt 16 Krankenhausneubauten bzw. grundlegende Renovierungen fließen. Am Ende dieses Prozesses wird Dänemark noch über 21 Akutkrankenhäuser verfügen, d. h. eins pro 265.000 Einwohner (Christiansen \& Vrangbaek 2018).

\section{Ergebnisse der Reform}

Nach der überwiegend bereits erfolgten Reduktion der Anzahl der Krankenhäuser und der Neuausrichtung der Notfallversorgung werden für Dänemark positive Auswirkungen auf die Versorgungsqualität berichtet. Dies verdeutlichen wesentliche Qualitätskennzahlen: So hat sich etwa die 30-Tage-Sterblichkeit nach akutem Herzinfarkt zwischen 2006 und 2015 von 7,2 auf 4 je 100 Patienten bei-

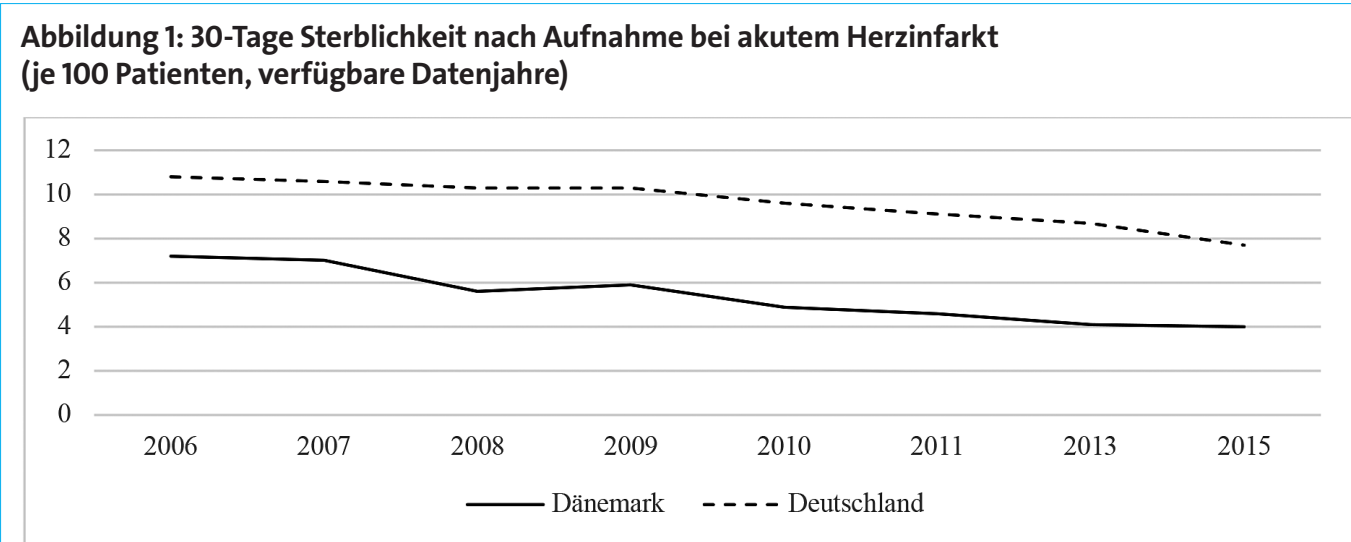

Quelle: Eigene Darstellung nach OECD 2018; Anmerkungen: alters-und geschlechtsstandardisierte Rate, ab 45 Jahre 


\section{Abbildung 2: OP innerhalb von 2 Tagen nach Aufnahme bei Hüftfraktur (je 100 Patienten, verfügbare Datenjahre)}

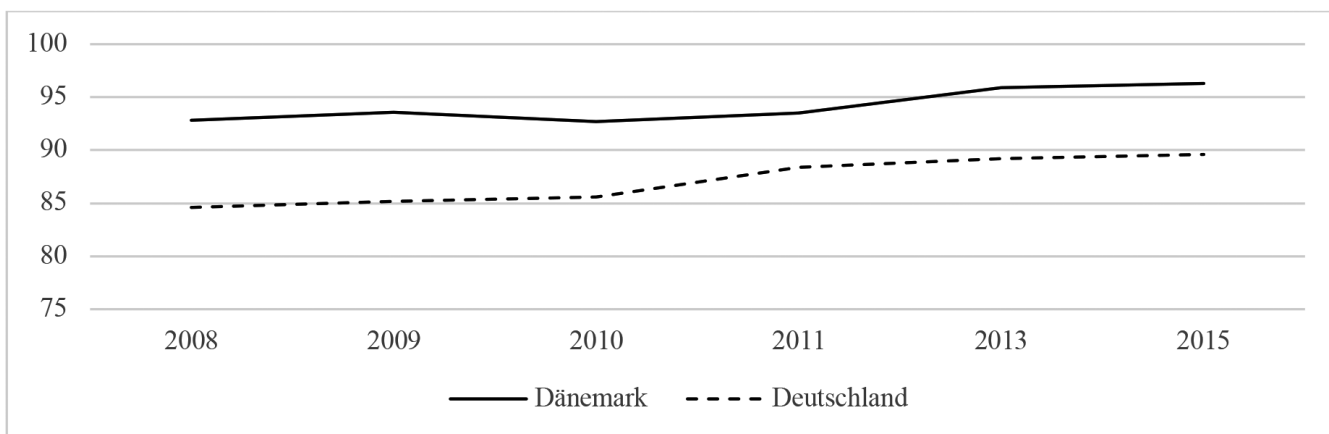

Quelle: Eigene Darstellung nach OECD 2018; Anmerkungen: rohe Rate, ab 65 Jahre gen, aber die Wegezeit sollte stets im Zusammenhang mit der Qualität der stationären Behandlung gesehen werden (Busse \& Berger 2018). So zeigen auch aktuelle Befragungen und Routinedatenanalysen aus Deutschland immer wieder, dass Patienten hierzulande für eine qualitativ hohe Versorgung ebenso bereits weitere Entfernungen als zum nächstgelegenen Krankenhaus in Kauf nehmen (BKK 2015). nahe halbiert. Deutschland konnte zwar im Vergleichszeitraum auch die Sterberate verringern, liegt aber heute noch klar über dem Niveau von Dänemark von vor über 10 Jahren (siehe Abb. 1). Trotz der deutlichen Reduktion der Krankenhäuser lassen sich sogar bei der Dauer bis zur Behandlung einer Hüftfraktur Verbesserungen verzeichnen (siehe Abb. 2). In Dänemark werden über $96 \%$ der Patienten innerhalb von 48 Stunden operativ versorgt. Dieser wichtige Prozessindikator wird auch im Rahmen der deutschen externen stationären Qualitätssicherung für das Aufdecken von strukturellen Defiziten eingesetzt und zeigt insbesondere, dass sich Krankenhauskapazitäten zum Wohle der Patienten konzentrieren lassen und damit der Zugang zu notwendigen medizinischen Maßnahmen verbessert werden kann. Dagegen zeigen sich in Deutschland trotz ebenfalls vorhandener Verbesserungen noch Werte, die deutlich unter dem dänischen Schnitt liegen (siehe Abb. 2). So werden hierzulande noch fast $11 \%$ der Patienten nicht zeitnah versorgt, was gerade bei älteren Patienten zum Selbständigkeitsverlust bis hin zur Pflegebedürftigkeit führen kann.

Auch bei der Betrachtung weiterer Schlüsselindikatoren zeigen sich im Zeitverlauf für Dänemark z. T. deutliche Verbesserungen (siehe Tab. 1). So hat sich etwa die durchschnittliche Verweildauer von 3,9 Tagen in 2007 auf 3,1 Tage in 2015 verkürzt. Auch bei der Wartezeit auf geplante chirurgische Eingriffe zeigen sich mit einer Reduktion von 66 Tagen auf 47 Tage zwischen 2009 und 2015 deutliche Verbesserungen. Ähnlich positive Entwicklungen gibt es auch bei anderen, eher übergeordneten, Indikatoren, wie dem allgemeinen Krankenstand der dänischen Bevölkerung.
Hinsichtlich der eingesetzten Ressourcen lässt sich feststellen, dass der Restrukturierungsprozess insgesamt ohne Abbau von Personal erreicht werden konnte. Im Gegenteil sind sogar mehr Fachkräfte als vor der Reform beschäftigt. Das ist nicht nur in absoluten Zahlen bemerkenswert, sondern wirkt sich bei einer Halbierung der Krankenhäuser direkt auf die Relation des pflegerischen und ärztlichen Personals pro Bett bzw. Fall und damit auf die Patientenversorgung aus (Christiansen \& Vrangbaek 2018). Kurzum: Die dänische Bevölkerung wird heute schneller mit höherem Personaleinsatz und besserer Qualität als noch zur Jahrtausendwende versorgt.

Zwar muss nun ein Teil der dänischen Bevölkerung etwas längere Wegezeiten für eine stationäre Behandlung bewälti-

\section{Umsetzung der Reform}

Bei einer derartig umfassenden Reform stellt sich jedoch nicht nur die Frage nach den Ergebnissen, sondern auch danach, wie diese konkret umgesetzt werden konnte. Daher werden folgend einige der Instrumente aufgeführt, die zur Umsetzung der Krankenhausstrukturreform in Dänemark eingesetzt worden sind.

Als eines der Schlüsselelemente kann dabei der Prozess der partizipativen Entscheidungsfindung gesehen werden. Unter Einbindung lokaler und regionaler Entscheidungsträger, klinischer Akteure und der Öffentlichkeit, wurde die Krankenhausplanung vollständig auf die nationale Ebene übertragen. Dort werden nun beispielsweise die Prioritäten für die Krankenhausversorgung allgemeingül-

Tabelle 1: Schlüsselindikatoren der regionalen Krankenhausstruktur und Leistung (2007-2015 oder nächstverfügbares Jahr)

\begin{tabular}{lll}
\hline & $\mathbf{2 0 0 7}$ & $\mathbf{2 0 1 5}$ \\
\hline Ärztliches Personal (Vollzeitäquivalent) & 13.109 & 15.632 \\
Pflegepersonal (Vollzeitäquivalent) & 31.472 & 35.622 \\
Sonstiges medizinisches Fachpersonal (Vollzeitäquivalent) & 23.057 & 23.263 \\
Beschäftigte insgesamt (Vollzeitäquivalent) & 67.637 & 74.516 \\
Anzahl an somatischen Betten & 15.835 & $13.299^{1}$ \\
Anzahl aufgenommener somatischer Patienten (in Tsd.) & 673 & 677 \\
Anzahl ambulante Patienten (in Tsd.) & 2.407 & 2.654 \\
Anzahl ambulante Besuche (in Tsd.) & 10.035 & 13.278 \\
Durchschnittliche Verweildauer (in Tagen) & 3,9 & $3,1^{2}$ \\
Anzahl chirurgischer Patienten (in Tsd.) & $508^{3}$ & $556^{4}$ \\
Wartezeit für geplante chirurgische Eingriffe (in Tagen) & $66^{5}$ & 47 \\
\hline
\end{tabular}

Quelle: Modifizierte Darstellung nach Christiansen \& Vrangbaek 2018; Anmerkungen: (1) Jahr 2014; (2) Jahr 2011; (3) Jahr 2008; (4) Jahr 2012; (5) Jahr 2009 
tig gesetzt. Dies und auch die objektiv kennzahlenbasierte Ermittlung der optimal anzustrebenden Krankenhausgröße sowie der Einbezug von verschiedenen Experten, hat ebenfalls zur Akzeptanz der Reform in der Bevölkerung beigetragen (Christiansen \& Vrangbaek 2018). Diese hat aktuellen Ergebnissen zufolge insgesamt ein deutlich höheres Vertrauen in das Gesundheitswesen als es etwa die deutsche Öffentlichkeit hat (OECD 2017b).

Neben vielen Verschiebungen von $\mathrm{Zu}$ ständigkeitsbereichen diverser Stakeholder und der Nutzung einer evidenzbasierten Planungsgrundlage ist das dänische Modell auch dadurch gekennzeichnet, dass die Krankenhauslandschaft dabei nicht isoliert betrachtet worden ist. Vielmehr wurde mit der Einführung einer elektronischen Patientenakte parallel auch die Digitalisierung im Gesundheitswesen vorangetrieben und eine Integration aller Versorgungsbereiche vorgenommen. Nicht zuletzt ist zu bemerken, dass wohl auch der langfristig angelegte Plan zum Strukturumbau, über Legislaturperioden hinweg, ein wichtiger Bestandteil des bisherigen Erfolgs der nach 10 Jahren noch andauernden Reform ist (Christiansen \& Vrangbaek 2018).

\section{Die dänische Reform als Impulsgeber}

Vor dem Hintergrund der oben beschriebenen Erfolge zeigen politische Entscheidungsträger und Akteure des Gesundheitswesens aus verschiedensten Ländern weltweit Interesse an dem dänischen Modell. Dies veranlasste die dänische Regierung zur Einrichtung von Healthcare DENMARK, einer gemeinnützigen Organisation, die Anfragen nunmehr gebündelt an die richtigen Ansprechpartner weiterleitet und vielfältige Informationsangebote zu den Krankenhausreformen bereitstellt (Healthcare DENMARK 2018). So trägt Healthcare DENMARK zur Weiterentwicklung des dänischen Gesundheitssystems und zur Förderung von dessen internationaler Sichtbarkeit bei. Dabei stehen die innovativen Gesundheitslösungen Dänemarks, insbesondere im Bereich des Krankenhaussektors im Mittelpunkt.

Healthcare DENMARK bietet einen Zugang zu einem breiten Netzwerk von Schlüsselfiguren der Politik, Wissenschaft und Versorgung. Viele internati- onale Akteure nutzten bereits die Möglichkeit, sich zu informieren - darunter z. B. aus Schweden, den Niederlanden und auch Deutschland sowie aus verschiedenen Ländern Nord- und Südamerikas (ebd.).

Insbesondere in Anbetracht des in Deutschland allgegenwärtig diskutierten Fachkräftemangels und einer zudem ähnlichen Bevölkerungsstruktur, Krankheitslast sowie Ausgangssituation kann das dänische Modell wichtige Impulse für die deutsche Krankenhauslandschaft geben. Denn um zukünftig die Versorgung auf einem hohen Qualitätsniveau zu sichern, steht diese vor immensen strukturellen Herausforderungen, insbesondere hinsichtlich der Kapazitätsund Leistungssteuerung. Der Planung und Organisation von Krankenhäusern kommt dabei eine entscheidende Rolle

\section{Internationale Impulse könnten helfen, Entwicklungsszenarien für eine umfassende Umgestaltung der deutschen Krankenhauslandschaft zu modellieren.}

zu, da mit ihr das Leistungsspektrum, das Einzugsgebiet und die Spezialisierung einzelner Standorte oftmals für Jahrzehnte festgeschrieben werden. Jedoch sind die bisherigen Bemühungen, den Herausforderungen zu begegnen äußerst fragmentiert, lokal begrenzt, statisch und zudem vom Dissens der Akteure des deutschen Gesundheitssystems geprägt. Dies äußert sich darin, dass statt einer umfassenden Reformierung nur vereinzelte Maßnahmenbündel eingesetzt werden, die wiederum nur zu einer punktuellen Konsolidierung der deutschen Krankenhauslandschaft beitragen. Dies zeigte sich auch zuletzt bei denen im Rahmen des Krankenhausstrukturgesetzes (KHSG) eingeführten Qualitätszu- und -abschlägen, den planungsrelevanten Qualitätsindikatoren oder auch den Qualitätsverträgen.

Internationale Impulse und Erfahrungen könnten helfen, diesen Prozess zu beschleunigen und Entwicklungsszenarien für eine umfassende Umgestaltung der deutschen Krankenhauslandschaft zu modellieren.
Dies wirft die Frage auf, inwieweit die dänische Krankenhausplanung als Vorbild für die Situation in Deutschland dienen kann bzw. welche Aspekte so spezifisch sind, dass sie nicht übertragbar sind. Adressiert wird diese Frage nun in einem Forschungsvorhaben, für das eine Förderung durch den G-BA-Innovationsfonds für Versorgungsforschungsprojekte beantragt wurde.

\section{Das Projekt Krankenhaus: Impulse für Deutschland aus Dänemark (K:IDD)}

Unter der Konsortialführung des Fachgebiets Management im Gesundheitswesen (MiG) der TU Berlin haben sich der BKK Dachverband, das Deutsche Krankenhausinstitut (DKI) und das Zentralinstitut für die kassenärztliche Versorgung $(\mathrm{Zi})$ zusammengefunden. Daneben konnten weitere Kooperationspartner gewonnen werden, wie Healthcare DENMARK, der Bundesverband Deutscher Privatkliniken (BDPK) e. V., das Ministerium für Arbeit, Gesundheit und Soziales des Landes Nordrhein-Westfalen, der BKK LandesverbandNordwest sowie eine Arbeitsgruppe der Fakultät für Gesundheitswissenschaften an der Universität Bielefeld, deren Expertise im Bereich der Analyse von Gesundheitspolitik und Gesundheitssystemen unter politikwissenschaftlichen Fragestellungen und mit politikwissenschaftlichen Methoden liegt.

Das kurzfristige Projektziel besteht in der Erarbeitung von Szenarien für die Versorgungslandschaft in Deutschland. Anhand einer detaillierten Analyse des dänischen Beispiels sollen Handlungsoptionen für eine Weiterentwicklung der Krankenhausversorgung und der sektorenübergreifenden Versorgung für Deutschland aufgezeigt und bewertet werden. Dies betrifft gleichermaßen die Versorgungsanalyse, etwa hinsichtlich technischer und medizinischer Kriterien wie Bedarfsgerechtigkeit, Erreichbarkeit, Qualität und Finanzierung sowie die Politikanalyse, d. h. die Fragen, welche Akteure bei der Reform in Dänemark eingebunden waren, wie der politische Prozess durchgeführt worden ist, welche Kriterien zugrunde gelegt worden sind und wie deren Einhaltung bewertet wurde. Mit den aus dem Projekt 
abgeleiteten Erkenntnissen sollen den relevanten gesundheitspolitischen Akteuren in Deutschland substantielle Handlungsalternativen zur Verbesserung der Patientenversorgung gegeben werden. Konkret sollen für den Gesetzgeber (bzgl. Ordnungsrahmen, institutioneller Ordnung und Aufgabenzuordnung, sektorenübergreifender Datenverfügbarkeit, Definition bundesweiter Rahmenplanungsvorgaben) und die untergesetzlichen bzw. landesgesetzlichen Normgeber (insb. den G-BA, die Länder hinsichtlich KH-Pläne/LandesKHG/ Förderungsbescheide sowie Partner der Selbstverwaltung auf Bundesebene und Landesebene/ Gremium 90a) entsprechende Vorschläge und Umsetzungshinweise bis hin zu konkreten Kodifizierungsvorschlägen erarbeitet werden. Diese sollen sich wiederum auf die ausgewählten Versorgungsaspekte gemäß den sekundären Forschungsfragen (wie Krankenhausplanung, Notfallversorgung, Zentrenbildung, Digitalisierung, Triagesysteme etc.) sowie die dafür erforderliche personelle, sachliche und finanzielle Ausstattung der Krankenhäuser beziehen. Langfristiges Projektziel ist es, die inhaltlichen und methodischen Voraussetzungen dafür zu schaffen, dass die übertragbaren Teile der dänischen Reformen in die deutsche Krankenhausversorgung integriert und für eine verbesserte Patientenversorgung genutzt werden können.

Das Forschungsvorhaben verwendet einen Methoden-Mix und ist in drei Arbeitspakete (AP) unterteilt: (1) eine Bestandsaufnahme der deutschen Krankenhauslandschaft und Vergleich mit dem dänischen Modell, (2) eine detaillierte Analyse der Reform in Dänemark und (3) die Übertragbarkeit der dort gewonnenen Erkenntnisse auf Deutschland.

In AP 1 ist zunächst eine umfassende Bestandsaufnahme der dänischen Gesundheits- und Krankenhausversorgung vorgesehen. Diese soll einerseits als Vergleich zur dänischen Ausgangssituation dienen und andererseits den Ausgangspunkt für die Analyse der Übertragbarkeit auf Deutschland darstellen. Die Ausarbeitung umfasst auch die Aspekte der zunehmenden Möglichkeiten für Krankenhäuser, Leistungen ambulant zu erbringen, ebenso wie die
Integration der Krankenhäuser in die Notfallversorgung. Damit wird insbesondere die Problematik des geteilten Sicherstellungsauftrages adressiert und die Analyse aus sektorenübergreifender Perspektive vorgenommen.

Methodisch kommen in diesem Arbeitspaket Literaturreviews, Dokumentenanalysen, statistische Sekundäranalysen sowie Experteninterviews mit

\section{Die vom dänischen Modell} gewonnenen Erkenntnisse önnen auch einen Beitrag zur Förderung er sektorübergreifenden Versorgung leisten.

Fachleuten aus Dänemark zum Einsatz. Daneben wird ein Workshop mit in der Krankenhausplanung/-finanzierung erfahrenen Praktikern aus Krankenhäusern, Verbänden und zuständigen Ministerien aus Deutschland durchgeführt. Dessen Ziel es ist, wesentliche Fragen und Bewertungskriterien für die Analyse und Übertragung des dänischen Modells zu generieren, die in AP 2 Berücksichtigung finden sollen.

Die Tiefenanalyse der dänischen Reform in AP2 umfasst die drei Aspekte Politik, Versorgung sowie praktische Umsetzung und erfolgt anhand verschiedener Techniken, zu denen Interviews mit wesentlichen Akteuren, Dokumentenanalysen von (wenn nötig übersetzten) Gesetzestexten, Verordnungen, Kommissionsunterlagen und Reviews zu wissenschaftlichen Evaluationen der dänischen Krankenhausreform gehören. Darüber hinaus wird auch eine mediale Rückschau (z. B. über Nachrichtenportale, Tageszeitungen, Fernsehbeiträge) durchgeführt um den öffentlichen Diskurs des Reformvorhabens nachzuzeichnen. Besonderer Fokus wird darauf gelegt, den Einbezug und Einfluss der wesentlichsten Interessensgruppen aus Politik, Medizin, Bürger- und Patientengruppen und Verbänden zu verstehen und Initiativen zu identifizieren, die eine derart tiefgreifende Reform ermöglicht haben. Für die Umsetzung dieses Arbeitspakets wurde bereits ein Netzwerk von dänischen Experten aufgebaut, welches im Rahmen von strukturierten Interviews,
Fokusgruppen, und Ad-hoc-Anfragen bei der Beantwortung der o. g. Fragestellungen unterstützend tätig sein wird. Dazu gehören Vertreter aus Politik, Institutionen, Versorgung und Wissenschaft. Als zentraler Anlaufpunkt wird Healthcare DENMARK dieses Netzwerk steuern und bei Bedarf weitere Experten hinzuziehen.

Im AP 3 sollen dann die Erfahrungen Dänemarks hinsichtlich der Übertragbarkeit auf das deutsche Gesundheitswesen untersucht werden. Diese Untersuchung beinhaltet zum einen weitergehende Fragestellungen zur Ähnlichkeit der Systeme vor der Reform (hinsichtlich Strukturen und Nutzung, aber auch Zuständigkeiten für Planung und Finanzierung) und auch die Frage, inwieweit die Probleme ähnlich gesehen werden (bzw. wurden) sowie die Auswertung der von den Krankenhausplanungsexperten in AP 2 genannten relevanten Fragestellungen.

Auch hier soll ein Methoden-Mix zum Einsatz kommen, zu dem eine Szenarioanalyse auf Grundlage von GKVRoutinedaten gehört. Dabei wird die Übertragbarkeit empirisch getestet und graduell modelliert um die Wirkung der in Dänemark angewandten Maßnahmen auf die deutsche Krankenhauslandschaft abzubilden. Im Rahmen dessen werden nicht nur die Erreichbarkeit, Wegezeiten und -strecken berücksichtigt, sondern auch international verfügbare Qualitätskriterien zur Leistungsplanung und steuerung einbezogen (u. a. Mindestmengen und Personaleinsatz). Um eine Bewertung aus allen im Gesundheitssystem relevanten Perspektiven zu ermöglichen, sind darüber hinaus auch Workshops mit Vertretern aller Akteure - darunter Bürger- und Patientenvertreter - und Experteninterviews vorgesehen.

\section{Ausblick}

Insbesondere durch den partizipativen Ansatz und die Einbindung aller relevanten Akteure können die Erkenntnisse des Forschungsvorhabens wichtige und dabei konkrete wie praktikable Handlungsempfehlungen für die notwendige Reformierung der Krankenhauslandschaft geben und so langfristig die Versorgung verbessern. Durch die Tiefenanalyse der dänischen Krankenhausstrukturreform und daraus resultierender Effekte können jene Bereiche identifiziert werden, in denen eine Versorgung der Patienten 
aus medizinischer, versorgungsplanerischer oder ökonomischer Sicht besser ist als in Deutschland. Die gleichzeitige Identifizierung der Bedingungen einer Übertragung durch den Austausch mit den relevanten Akteuren ermöglicht das Aufzeigen von Wegen, die zu eben solchen Verbesserungen führen können, indem sie an den deutschen Kontext und Versorgungsalltag angepasst sind. Dabei werden auch und insbesondere gesetzliche und untergesetzliche Normgeber (G-BA) sowie die Partner der Selbstverwaltung adressiert, denen durch die
Szenarioanalyse konkrete Lösungsansätze gegeben werden. Die Digitalisierung der Krankenhäuser ist in Dänemark weitaus deutlicher fortgeschritten als in Deutschland. Das Projekt wird auch diesbezügliche Rahmenbedingungen, Voraussetzungen und erforderliche Prozesse in Dänemark ermitteln, woraus sich Erkenntnisse für die erforderliche Digitalisierung deutscher Krankenhäuser ziehen. Die Rahmenbedingungen, Voraussetzungen und Konsequenzen einer Anpassung der Krankenhauskapazitäten in Dänemark werden ausführlich ana- lysiert und im Hinblick auf die Folgen für die Versorgung diskutiert. Dadurch können spezifische Reformvorschläge für die deutsche Versorgungslandschaft generiert werden. Diese Ergebnisse können für die Politikberatung greifbar gemacht werden. Da das dänische Modell durch eine ganzheitliche Betrachtung der Gesundheitsversorgung gekennzeichnet ist, betreffen die gewonnenen Erkenntnisse nicht ausschließlich den stationären Sektor, sondern können auch einen Beitrag zur Förderung der sektorübergreifenden Versorgung leisten.

\section{Literatur}

Augurzky, B.; Beivers, A.; Straub, N.; Veltkamp C. (2014): Krankenhausplanung 2.0. Endbericht zum Forschungsvorhaben des Verbandes der Ersatzkassen e. V. (vdek). Hg. v. Rheinisch-Westfälisches Institut für Wirtschaftsforschung. Essen (RWI Materialen, Heft 84).

Augurzky, B.; Beivers, A.; Giebner, M.; Kirstein, A. (2015): Organisation der Notfallversorgung in Dänemark: Lösungsansätze für deutsche Probleme? In: J. Klauber, M. Geraedts, J. Friedrich und J. Wasem (Hg.): KrankenhausReport 2015. Schwerpunkt: Strukturwandel. Stuttgart: Schattauer, S. 77-98.

Busse, R.; Ganten, D.; Huster, S.; Reinhardt, E. R.; Suttorp, N.; Wiesing, Urban (2016): Zum Verhältnis von Medizin und Ökonomie im deutschen Gesundheitssystem. 8 Thesen zur Weiterentwicklung zum Wohle der Patienten und der Gesellschaft. Diskussion Nr. 7. Hg. v. Deutsche Akademie der Naturforscher Leopoldina - Nationale Akademie der Wissenschaften. Halle (Saale) (Diskussionspapiere). Online verfügbar unter https://www.leopoldina.org/ uploads/tx leopublication/Leo Diskussion Medizin_und_Oekonomie_2016.pdf, zuletzt geprüft am 25.10.2017.

BKK (2015): BKK Umfrage 2015. Chronisch Kranke. Krankenhausversorgung. Online verfügbar unter https://www.bkk-dachverband.de//fileadmin/user_upload/Anlage_PM_23_9_2015
Umfrage_Chronisch_krank_Krankenhaus.pdf, zuletzt geprüft am 27.10.2017.

Busse, R.; Berger, E. (2018): Weniger (Standorte, Betten und Fälle) ist mehr (Zugang, Qualität und Ergebnisse): Standpunkte der Gesundheitsökonomie. In: Janssen, D.; Augurzky, B. (Hg.): Krankenhauslandschaft in Deutschland. Zukunftsperspektive - Entwicklungstendenzen - Handlungsstrategien. Stuttgart Kohlhammer, im Druck.

Christiansen, T.; Vrangbaek, K. (2018): Hospita centralization and performance in Denmark Ten years on. Health Policy 122(4), S. 321-328.

Christiansen, T. (2012): Ten years of structural reforms in Danish healthcare. In: Health Policy 106(2), S. 114-119.

Geissler, A.; Wörz, M.; Busse, R. (2010): Deutsche Krankenhauskapazitäten im internationalen Vergleich. In: J. Klauber, J.; Geraedts, M. Friedrich, J. (Hg.): Krankenhaus-Report 2010. Schwerpunkt: Krankenhausversorgung in der Krise? Stuttgart: Schattauer, S.25-40.

Healthcare DENMARK (2018): Delegation visits. Online verfügbar unter http://healthcaredenmark.dk/, zuletzt geprüft am 25.04.2018.

Nimptsch, U.; Mansky, T. (2017): Hospital volume and mortality for 25 types of inpatient treatment in German hospitals: observational study using complete national data from
2009 to 2014. In: BMJ open 7(9), e016184. DOI 10.1136/bmjopen-2017-016184.

OECD (2017a): Health at a Glance 2017. OECD Indicators. OECD. Online verfügbar unter https://read.oecd-ilibrary.org/social-issuesmigration-health/health-at-a-glance-2017 health_glance-2017-en\#page1, zuletzt geprüft am 18.05.2018.

OECD (2017b): Tackling wasteful Spending on Health. Highlights. OECD. Online verfügbar unter https://www.oecd.org/els/healthsystems/Tackling-Wasteful-Spending-onHealth-Highlights-revised.pdf, zuletzt geprüft am 16.05.2018.

OECD (2018): Health Care Quality Indicators Patient Safety. Online verfügbar unter http:// www.oecd.org/els/health-systems/hcqi-patient-safety.htm, zuletzt geprüft am 16.05.2018.

Olejaz, M.; Nielsen, A.; Rudkjøbing, A.; Okkels Birk, H; Krasnik, A.; Hernández-Quevedo, C. (2012): Denmark: Health system review. Health Systems in Transition 14(2).

Statistisches Bundesamt (2016a): Kostennachweis der Krankenhäuser. Wiesbaden. Online verfügbar unter www.gbe-bund.de, zuletzt geprüft am 25.04.2018.

Statistisches Bundesamt (2016b): Krankenhausstatistik - Grunddaten. Wiesbaden. Online verfügbar unter www.gbe-bund.de, zuletzt geprüft am 25.04.2018 